\title{
Identifying Risk for Social/Emotional Difficulty in Adolescents with Sickle Cell Disease Using the Pediatric-Symptom-Checklists
}

\author{
Susumu Inoue ${ }^{1,2^{*}}$, Crystal Cederna Meko Psy $D^{1,2}$, Tammy Scherrer RN ${ }^{1}$ and Jenny LaChance MS ${ }^{3}$ \\ ${ }^{1}$ Department of Pediatrics, Hurley Medical Center/Hurley Children's Hospital, Flint, MI, USA \\ ${ }^{2}$ Department of Pediatrics/Human Development, Michigan State University College of Human Medicine, Flint, MI, USA \\ ${ }^{3}$ Department of Research, Hurley Medical Center, Flint, MI, USA \\ *Corresponding author: Susumu Inoue, Department of Pediatrics Hurley Medical Center, Flint, MI, 48503, USA, Tel: (810) 513-7600; Fax: (810) 252-9972; E-mail: \\ sinoue1@hurleymc.com
}

Received date: March 17, 2017; Accepted date: March 31, 2017, Published date: April 05, 2017

Copyright: (c) 2017 Inoue S, et al. This is an open-access article distributed under the terms of the Creative Commons Attribution License, which permits unrestricted use, distribution, and reproduction in any medium, provided the original author and source are credited.

\begin{abstract}
The Pediatric Symptom Checklist (PSC) is used to screen for social/emotional difficulty in youth. It is completed by either a parent (PSC) or patient (Y-PSC). The Achenbach Child Behavior Checklist (CBCL) and Youth Self-Report (YSR) are parent- and self-completed social/emotional assessment tools that are well validated, but the less validated PSC and Y-PSC's availability, convenience, and cost are advantageous for busy clinics. Objective: We examined the appropriateness of using the PSC and Y-PSC to screen adolescents with sickle cell disease for social/ emotional difficulty. Method: Patients with sickle cell disease $(n=14 ; 7$ female; mean age $\pm S D=4.1 \pm 1.8)$ completed the Y-PSC and YSR, while their parents completed the PSC and CBCL. Rates of risk for social/emotional difficulty were compared between parent-completed forms (PSC versus CBCL), and between patient-completed forms ( $Y$ PSC and YSR). Agreement in the presence or absence of risk was explored as well. Results: Six youth had total scores exceed cutoffs for a positive screen. Parent-completed questionnaires each identified 4 youth; the YSR and Y-PSC identified 4 and 2 respectively. However, no 2 tools identified the exact same combination of youth and no single informant or tool captured all youth with risk for social/emotional difficulty. Conclusion: Our results, though preliminary, indicate that no single informant or screening tool sufficiently captures risk for social/emotional difficulty in the adolescent sickle cell population. Instead, a multi-informant, multi-method approach to screening for social/ emotional difficulty in adolescents with sickle cell disease is encouraged until additional research can illuminate an alternative.
\end{abstract}

Keywords: Screen for social and emotional difficulty; Sickle cell disease; Adolescents; Pediatric symptom checklist

\section{Introduction}

Children with sickle cell disease suffer from repetitive severe pain episodes due to occlusion of capillary vessels and postcapillary venules. Occlusion occurs when red blood cells containing sickle hemoglobin transform into a highly rigid, non-deformable state under deoxygenated condition. In addition to episodic pain, youth experience progressive organ damage as they get older, and have a short lifespan, generally limited to about 60 years [1]. Because of the impact of illness upon functioning, many patients with sickle cell disease are depressed, and this is amply documented in the literature $[2,3]$.

The Pediatric Symptom Checklist (PSC) is a 1-page questionnaire designed to screen for risk of social/emotional difficulty in school-age children [4,5]. It has 2 versions: a form to be completed by parents or caregivers of children ages 6-18 years old (PSC) [6] and a form to be completed by adolescents ages 11 years and older (self-report) (Y-PSC) $[7,8]$. Each form takes about 5 minutes to complete. Most research has been done with the PSC (parent) version 9 and suggests satisfactory validity in a variety of patient populations, including an inner-city population [9] and those with thalassemia [10], sickle cell disease, insulin-dependent diabetes mellitus, [5] or dermatological disorders [11]. Despite preliminary validation, the aforementioned studies did not establish predictive or criterion-related validity using wellestablished social/emotional questionnaires. Consequently, the applicability of the general population norms to various subpopulations, such as those with sickle cell disease, remains unclear [8]. The Y-PSC (youth) has been used less often and lacks validation in youth with chronic illnesses.

The Achenbach System of Empirically Based Assessment (Achenbach), specifically the Child Behavior Checklist (CBCL) and Youth Self-Report (YSR) [12], has been widely used and well validated to screen for social/emotional difficulty in adolescents. The CBCL is completed by parents, while the YSR is completed by patients themselves. Both are lengthy, each consisting of over 100 items. Each form usually takes about 30 minutes to complete and requires hand or computerized scoring [13]. Relative to the PSC and Y-PSC, the CBCL and YSR are more costly, take longer to complete, and require additional training and user qualifications. Thus, the CBCL and YSR are less practical and less likely to be used in a busy clinic where the goal of screening is to detect risk for social/emotional difficulty.

In this study, we aimed to examine the ability of the PSC and Y-PSC to detect risk for social/emotional difficulty at a rate similar to the CBCL and YSR respectively. Additionally, we explored overall agreement between the PSC and CBCL, as well as between the Y-PSC and YSR in their assessment of risk for social/emotional difficulty in adolescents with sickle cell disease. We reasoned that if the PSC and YPSC detected risk for social/emotional difficulty sufficiently, as determined by agreement in the presence or absence of risk when 
compared to the parallel Achenbach assessment tool, then adoption of the PSC and Y-PSC for such screening could be considered.

\section{Materials and Methods}

Our target population was adolescents with sickle cell disease. Subjects were recruited from our pediatric comprehensive sickle cell clinic, a clinic serving 6 counties in a Midwestern state. For the patients to be included in this study, they had to be (1) accompanied by a parent or legal guardian, (2) 11 to 18 years old, and (3) seen regularly at our sickle cell clinic for one form of sickle cell syndrome, i.e., homozygous sickle cell disease, sickle cell/beta thalassemia, or double heterozygous hemoglobin $\mathrm{S}$ and hemoglobin $\mathrm{C}$ disease.

Both the patients and parents or legal caregivers were asked to participate in this study. A dedicated clinic nurse asked them to review and sign the consent/assent forms. Once they signed their respective consent/assent forms, the patients and parents were separated. The patients were given the Pediatric Symptom Checklist youth self-report (Y-PSC) questionnaire and Youth Self-Report ages 11-18 (YSR). The parents were given the Pediatric Symptom Checklist (PSC) questionnaire and Child Behavior Checklist ages 6-18 (CBCL). For the CBCL and YSR, we asked each subject to complete questions on pages 3 and 4 only (i.e., only items factoring into the social/emotional domain scores).
A pediatric psychologist received the completed questionnaires, without identifiable information, hand scored the PSC and Y-PSC assessment tools, and computer scored the CBCL and YSR assessment tools using the test publisher's software program developed for this purpose.13 Scores were computed for each assessment tool (overall score) and subdomain (internalizing, externalizing, and attention problems). These scores were used to determine risk for social/ emotional difficulty, as described in Table 1 , and were dichotomized into "positive screen" or "negative screen" according to the following:

Positive Screen: A score at or above the domain's published threshold.

Negative Screen: A score below the domain's published threshold.

Rates of risk for social/emotional difficulty were compared between parent-completed forms and patient-completed forms. Agreement in the presence or absence of risk was explored as well. Data were analyzed using the statistical package for the social sciences (SPSS 15.6, 2006). Correlative data were analyzed by calculating the Pearson correlation coefficient ( $r$ ). Alpha $<0.05$ (2-tailed) was defined as statistically significant.

This investigation was approved by our institutional review board.

\begin{tabular}{|l|l|l|l|l|}
\hline & $\begin{array}{l}\text { Overall Score } \\
\text { (Total Problems) }\end{array}$ & Internalizing Subdomain & Externalizing Subdomain & Attention Problems Subdomain \\
\hline CBCL \& YSR & $\geq 60$ (T-score) & $\geq 60$ (T-score) & $\geq 60$ (T-score) & $\geq 65$ (T-score) \\
\hline PSC \& Y-PSC & $\begin{array}{l}\geq 28 \text { (PSC) } \\
\geq 30 \text { (Y-PSC) }\end{array}$ & $\geq 5$ (raw score) & $\geq 7$ (raw score) & $\geq 7$ (raw score) \\
\hline
\end{tabular}

Positive scores indicate risk for social/emotional difficulty. CBCL, Child Behavior Checklist; YSR: Youth Self-Report; PSC: Pediatric Symptom Checklist; Y-PSC: Pediatric Symptom Checklist youth self-report.

Table 1: Definitions of positive scores for each tool and subdomain.

\section{Results}

Subjects were 14 parent-child pairs consisting of patients with sickle cell disease whose ages ranged from 11 to 18 years (mean=14.1; standard deviation=1.8). Of the 14 patients ( 7 females), 8 had hemoglobin SC disease, 5 had homozygous sickle cell disease, and 1 had $\mathrm{Hb} S / \beta$-thalassemia. Thus there was an overrepresentation of the $\mathrm{Hb}$ SC disease.

\section{Risk for social/emotional difficulty}

In terms of their overall scores (not subdomain scores), a total of 6 participants were screened positive by at least 1 of the 4 instruments (Figure 1). The Child Behavior Checklist (CBCL), Pediatric Symptom Checklist (PSC), and Youth Self-Report (YSR) each yielded 4 subjects with risk for social/emotional difficulty. However, these 3 screening tools did not identify the exact same 4 participants; each tool missed 2 subjects who were picked up by at least 1 of the others. The Pediatric Symptom Checklist youth self-report (Y-PSC) yielded only 2 positive screens, and is the least sensitive tool.

Reviewing the 3 subdomains separately, as seen in Figure 1, a total of 8 participants had at least 1 positive screen in the internalizing subdomain. CBCL scores appeared to result in the most positive screens (i.e., 6 positive screens) in this area, followed by YSR scores, which yielded 5 positive screens. The Y-PSC returned the fewest positive screens (i.e., 1 positive screen) here. Interestingly, there were only 4 subjects who screened positive in both the externalizing and attention subdomains. In each of these areas as well, the Y-PSC rendered only 1 positive screen.

\section{Sensitivity of each instrument}

Looking at overall scores, the parent-completed questionnaires identified subjects with risk for social/emotional difficulty at the same rate, while the patient-completed forms did not. Although there was good statistical agreement between the CBCL and PSC $(r=0.854$; alpha $=-0.000)$ as well as between the YSR and Y-PSC $(r=0.658$; alpha=-0.110), the clinical agreement was insufficient for both informant groups. Parent-completed forms each missed 1 youth picked up by the other one. As for the patient-completed forms, there was only 1 subject who was captured by both. No 2 tools detected the same combination of youth and no single informant or screening tool captured all 6 subjects with risk for social/emotional difficulty. One youth was identified as at risk by all 4 instruments, 1 by 3 instruments, 3 by 2 instruments, and 1 by only 1 instrument. 
Citation: Inoue S, Meko Psy DCC, Scherrer RNT, LaChance MSJ (2017) Identifying Risk for Social/Emotional Difficulty in Adolescents with Sickle Cell Disease Using the Pediatric-Symptom-Checklists. J Blood Disord Transfus 8: 377. doi:10.4172/2155-9864.1000377

Page 3 of 4

\begin{tabular}{|c|c|c|c|c|}
\hline Pair No. & $\begin{array}{c}\text { Overall } \\
\text { (Total Score) }\end{array}$ & $\begin{array}{l}\text { Internalizing } \\
\text { Subdomain }\end{array}$ & $\begin{array}{c}\text { Externalizing } \\
\text { Subdomain }\end{array}$ & $\begin{array}{c}\text { Attention Problems } \\
\text { Subdomain }\end{array}$ \\
\hline \multicolumn{5}{|l|}{101} \\
\hline \multicolumn{5}{|l|}{102} \\
\hline \multicolumn{5}{|l|}{103} \\
\hline \multicolumn{5}{|l|}{104} \\
\hline \multicolumn{5}{|l|}{105} \\
\hline \multicolumn{5}{|l|}{106} \\
\hline \multicolumn{5}{|l|}{107} \\
\hline \multicolumn{5}{|l|}{108} \\
\hline \multicolumn{5}{|l|}{109} \\
\hline \multicolumn{5}{|l|}{110} \\
\hline \multicolumn{5}{|l|}{111} \\
\hline \multicolumn{5}{|l|}{112} \\
\hline \multicolumn{5}{|l|}{113} \\
\hline \multicolumn{5}{|l|}{114} \\
\hline \multicolumn{5}{|c|}{ A filled square shows a positive screen. A blank square shows a negative screen. } \\
\hline & \multicolumn{4}{|c|}{$=$ positive Pediatric Symptom Checklist screen } \\
\hline & \multicolumn{4}{|c|}{$=$ positive Youth Self-Report screen } \\
\hline & \multicolumn{4}{|c|}{ = positive Pediatric Symptom Checklist youth self-report screen } \\
\hline
\end{tabular}

Figure 1: Positive screens for risk of social/emotional difficulty designated by screening tool and domain.

\section{Discussion}

To improve outcomes for youth with sickle cell disease, early identification and treatment of social/emotional difficulty is essential. Psychosocial factors, rather than biomedical factors, have been shown to play a major role in adolescents' adaptability to sickle cell disorders [14]. That is, well-adapted teens have less depression and anxiety.

Numerous social/emotional assessment tools are available, but those that are well-validated, such as the Child Behavior Checklist, often are costly, time consuming, and require additional training. These factors limit the utility of social/emotional screening in busy clinics, despite the recognized importance of detecting and addressing social/ emotional difficulty. The Pediatric Symptom Checklist (PSC) and Pediatric Symptom Checklist youth self-report (Y-PSC) initially appeared to be the ideal alternatives to the gold standard screening tools for their efficiency and accessibility. They are easily integrated into the workflow of busy clinics because they are brief, free to use, and require little additional training. Furthermore, the PSC and Y-PSC are easy to score and interpret. The official recommendations attached to the Y-PSC form are that if the overall score is $\geq 30$ or if the answer to questions 36 or 37 (current or past suicidal ideation) is yes, then the patient should be evaluated carefully or referred to a mental health specialist. The PSC (not Y-PSC) uses a cut-off of $\geq 28$ to suggest risk for social/emotional difficulty. For clinics wishing to explore specific concerns, the PSC and Y-PSC also have easy-to-score subdomains.

In our earlier clinical experiences with 14 subjects with sickle cell disease (not a part of this study, and different subjects) who routinely visited our clinic, only 1 patient scored over 30 on the Y-PSC, fewer than expected (unpublished observation). Extensive literature searches revealed no studies evaluating the use of the Y-PSC in patient populations with chronic medical conditions, such as ours.

With regard to overall scores, this present study showed the Y-PSC identified only 2 of 6 subjects who were identified by at least 1 of the screening tools, and only 1 of 4 subjects detected by the parallel patient-completed tool. However, none of the 4 instruments captured all 6 potentially troubled youth. Thus, our results indicate that none of these tools, used alone, captured all youth with risk for social/ 
Citation: Inoue S, Meko Psy DCC, Scherrer RNT, LaChance MSJ (2017) Identifying Risk for Social/Emotional Difficulty in Adolescents with Sickle Cell Disease Using the Pediatric-Symptom-Checklists. J Blood Disord Transfus 8: 377. doi:10.4172/2155-9864.1000377

Page 4 of 4

emotional difficulty who would benefit from additional evaluation and/or referral to mental health providers. The same holds true for the 2 informant groups; neither detected all 6 subjects with positive screens. The results also indicate that the Y-PSC was least likely to identify risk for social/emotional difficulty.

Our sample population was overly represented by patients with hemoglobin SC disease. These patients generally present with fewer pain episodes than patients with homozygous sickle cell disease, though there are numerous exceptions. Thus the result may not apply to children with homozygous sickle cell disease.

Due to this study's small sample size and the absence of a healthy, demographically matched control group, it is difficult to generalize the results. Despite these limitations, our study finds strength in being the first to compare PSC and Y-PSC results with gold standard social/ emotional assessment tools in this patient population. As such, it offers a preliminary view into the way busy clinics may wish to screen for risk of social/emotional difficulty in adolescents with sickle cell disease. Although less efficient, our results encourage a multi-method, multiinformant approach to screening for social/emotional difficulty.

\section{Conclusions}

Our results did not support any 1 instrument as advantageous to another in detecting risk for social/emotional difficulty. Although tentative due to the small sample size, a multi-informant, multimethod approach to screening for social/emotional difficulty in youth with sickle cell disease is encouraged. Study replication with a larger sample that includes a control group is desirable.

\section{Acknowledgement}

The authors extend their sincere thanks to Katherine Negele for her critical comments and editing of this manuscript.

\section{References}

1. Elmariah H, Garrett ME, De Castro LM (2014) Factors associated with survival in a contemporary adult sickle cell disease cohort. Am J Hematol 89: 530-535.
2. Mahdi N, Al-Ola K, Khalek NA (2010) Depression, anxiety, and stress comorbidities in sickle cell anemia patients with vaso-occlusive crisis. J Pediatr Hematol Oncol 32: 345-349.

3. Yang YM, Cepeda M, Price C (1994) Depression in children and adolescents with sickle-cell disease. Arch Pediatr Adolesc Med 148: 457460.

4. Jellinek M, Little M, Murphy JM (1995) The Pediatric Symptom Checklist. Support for a role in a managed care environment. Arch Pediatr Adolesc Med 149:740-746.

5. Stoppelbein L, Greening L, Jordan SS (2005) Factor analysis of the Pediatric Symptom Checklist with a chronically ill pediatric population. J Dev Behav Pediatr 26: 349-355.

6. Massachusetts General Hospital (2017) Pediatric symptom checklist: Scoring the PSC.

7. Pagano ME, Cassidy LJ, Little M (2000) Identifying psychosocial dysfunction in school-age children: The Pediatric Symptom Checklist as a self-report measure. Psychol Sch 37: 91-106.

8. Massachusetts General Hospital (2017) Pediatric Symptom Checklist: Research.

9. Murphy JM, Reede J, Jellinek MS (1992) Screening for psychosocial dysfunction in inner-city children: Further validation of Pediatric Symptom Checklist. J Am Acad Child Adolesc Psychiatry 31: 1105-1111.

10. Saini A, Chandra J, Goswami U (2007) Case control study of psychosocial morbidity in beta thalassemia major. J Pediatr 150: 516-520.

11. Rauch PK, Jellinek MS, Murphy JM (1991) Screening for psychosocial dysfunction in pediatric dermatology practice. Clin Pediatr 30: 493-497.

12. Achenbach TM, Rescorla LA (2001) Manual for the ASEBA school-age forms and profiles. Burlington, VT: University of Vermont, Research Center for Children, Youth, and Families.

13. Achenbach $\mathrm{T}$ (1991) Manual for the child behavior checklist/4-18 and the 1991 profile. Burlington, VT: University of Vermont, Department of Psychiatry.

14. Burlew K, Telfair J, Colangelo L (2000) Factors that influence adolescent adaptation to sickle cell disease. J Pediatr Psychol 25: 287-299. 